\title{
Actual situation of the weight control in the Japanese functional indication food and food for specified health use
}

\begin{abstract}
A system using food for improvement in mild obesity and glycometabolism in individuals with borderline metabolic disorder exists in Japan. Because functionality of a product can be advertised assuming confirmation of its effect in humans, the market for such products has tended to increase. Several functional ingredients are known to originate from natural products, and their mechanisms of action against obesity are thought to involve lipid lowering and lipid utilization efficiency. Improvement in glycometabolism is thought to involve inhibition of glucose absorption in the small intestine or increased phosphorylation of the insulin receptor. Several functional ingredients are common food components, and several of them are known to be safe. However, their interactions with medications and effects of excessive consumption are unknown, and obtaining the relevant safety information is essential.
\end{abstract}

Keywords: weight control, Visceral fat, body fat, Japanese functional indication food, food for specified health use
Volume 8 Issue 2 - 2018

\begin{abstract}
Ryuji Takeda,' Akiyoshi Sawabe ${ }^{2}$
'Department of Nutritional Sciences for Well-being, Faculty of Health Sciences for Welfare, Kansai University of Welfare Sciences, Japan

2Department of Applied Biological Chemistry, Kindai University, Japan
\end{abstract}

Correspondence: Ryuji Takeda, Department of Nutritional Sciences for Well-being, Faculty of Health Sciences for Welfare, Kansai University of Welfare Sciences, 82-0026 Asahigaoka 3-III, Kashiwara city Osaka, Japan, Email rtakeda@tamateyama.ac.jp

Received: January 30, 2018 | Published: March 14, 2018

\section{Introduction}

Controlling obesity, which is one of the risk factors leading to diabetes and heart problems, is important. It is desirable to promote an improvement in this condition via appropriate dietary management and moderate exercise. However, modern social life makes consistent dietary management and exercise challenging. The number of obese individuals in Japan has been increasing, and is of particular concern among working populations aged 30 years or older. Increase in social security budgets including medical costs for the elderly has become a serious problem, and the Japanese Government has called for the prevention of life-style related diseases such as obesity1. However, several generations of working populations have been unable to continue dietary management and moderate exercise consistently. Therefore system using food for specified health purposes and functional indication foods for obesity improvement has been established. The major difference between food for specified health use and functional indication foods is that the former needs national agency approval and the latter does not. Foods for specified health use are foods authorized based on national-level evaluation and functional indication foods are those that are reported to meet the criteria of scientific basis of functionality. We cannot claim to heal and prevent diseases using this system of food, as we can with medications. However, in individuals at the borderline of health and disease, the beneficial effect of food on mild obesity and mild fasting blood sugar can be seen. Thus, treatment of individuals with disease is done using medications, and treatment of borderline individuals is done using functional food and improvement in lifestyle, preventing progression to disease. Functional ingredients in food which are known to cause obesity improvement and improvement in fasting blood sugar are mostly constituents found in natural products. In this review, we introduce functional ingredients in food and their functions.

\section{Weight control and suppression of body fat}

An important guideline for obesity improvement in Japan is visceral fat and body fat around the navel measured via BMI and CT scanning. ${ }^{1}$ Because it is necessary to clearly differentiate the role of medications and food as described earlier, clinical trials are performed in individuals with $25 \leqq \mathrm{BMI}<30$ corresponding to pre-obesity in the $\mathrm{WHO}^{2}$ criteria, and the products are made available as functional foods. Because such foods attract attention of consumers in the middle and old age groups, they tend to purchase them significantly. For example, green tea containing the glycoside quercetin and tea containing catechin are sold as functional ingredients included in a product authorized as a specified health food. Tea catechins have been confirmed to cause a decrease in body fat in humans. ${ }^{3}$ Regarding other possible mechanisms of action of fat decrease, ${ }^{4}$ lipolysis effects have been confirmed in an experiment using mouse 3T3-L1 cells. Further, tea catechin increased liver fat beta-oxidation in mice fed a high-fat diet for one month ${ }^{5}$ and promoted increased fat utilization. Similarly, the glycoside quercetin has been shown to decrease body fat in humans. ${ }^{6}$ Other functional ingredients included in products sold as functional indication foods based on confirmation of body fat decrease in humans are Glabridin from licorice, ${ }^{7}$ isoflavone extracted from the flower of Pueraria montan, ${ }^{8}$ apple polyphenol, ${ }^{9}$ and lactic acid bacteria. ${ }^{10}$ Their mechanisms of action mainly involve increased fat utilization such as the promotion of liver beta-oxidation and lipid lowering effects. ${ }^{11,12}$

In addition, several foods showing inhibitory effects on postcibal blood triglyceride levels in humans and inhibition of pancreatic lipase are being sold. For example, food including polyphenols from oolong tea ${ }^{13}$ globin protein split-product (Globin digest (GD) prepared from globin via acidic protease treatment),${ }^{14}$ and low-digestibility dextrin 
have shown confirmed effects in humans and are being sold as functional ingredients.

\section{Improvement in blood glucose levels}

The occurrence of diabetes in association with obesity is high and may lead to vascular disorder and renal failure. The main subjects of foods for specified health use and functional indication foods are people with fasting blood sugar in the range of $110 \mathrm{mg}-125 \mathrm{mg} /$ dL. Active functional food products decrease fasting blood sugar and tend to increase depression and food intake, and thus postcibal blood glucose. The functional ingredients in these products include low-digestibility dextrin, albumin from wheat, ${ }^{15}$ and polyphenols and $\mathrm{L}$-arabinose ${ }^{16}$ from leaves of the guava, ${ }^{17}$ which are known to inhibit blood glucose after a meal. Regarding their mechanisms of action, low-digestibility dextrin slows the absorption of sugar in the small intestine, guava leaf polyphenols inhibit alpha-amylase in the small intestine, and L-arabinose inhibits saccharase. For reduction in fasting blood sugar via long-term consumption, a product containing the functional ingredients corosolic acid and 5-aminolevulinic acid ${ }^{18}$ from the leaves of Banaba (Lagerstroemia speciosa) ${ }^{19}$ are sold. The mechanism of action of corosolic acid is known to be promotion of sugar uptake by enhancing phosphorylation of the insulin receptor. ${ }^{20}$ The detailed mechanism of 5-aminolevulinic acid action is unknown, although it is thought to increase cytochromec oxidase activity, contributing to improvement in carbohydrate metabolism.

Maintenance of appropriate diet and moderate exercise are primarily necessary for improvement in obesity and glycometabolism and prevent the development of various clinical conditions. However, such practice is often challenging in modern social life. Obesity in old and middle age groups is becoming a serious problem in Japan and is recognized as a cause for future cardiovascular problems. Similarly, improper glycometabolism is a major risk factor for nephropathy. Further, these conditions contribute to increased burden on the working population and increase in social security budgets including medical care, which are serious social problems. It is important that we control our own health appropriately to prevent disease. Further, it is important to use alternative therapies such as functional foods, using them for improving obesity and management of blood glucose. We have established systems of functional foods in Japan, distinct from that of medications. These systems are very likely to be sustained and related product development is likely to increase in the future. The health industry in Japan continues to grow, and development of functional ingredients based mainly on natural products is expected to increase. However, information regarding such products including safety information is not sufficiently available, and while most individuals rely on the fact that they are present in food as a basis for their safety, there is little information regarding their interactions with medications or effects of excessive consumption. Development of various functional food ingredients is expected in the future, but details regarding their functionality as well as safety information will be required.

\section{Conclusion}

In Japan, a system using functional foods has been developed to enable slight improvements in obesity and glycometabolism in individuals with borderline disease. Their functional ingredients include several natural products known to show effects in humans. The scientific basis for their actions and safety is relatively unknown. However, this area is expected to grow in the future because these ingredients are regarded as safe due to their presence in food

\section{Acknowledgments}

None.

\section{Conflicts of interest}

None.

\section{References}

1. Minister of Health, Labour and Welfare. Government of Japan; 2012.

2. WHO. Physical status: the use and interpretation of anthropometry. Report of a WHO Expert Committee. World Health Organ Tech Rep Ser. 1995;854:1-452.

3. Tsuchida R. Reduction of Body Fat in Humans by Long-term Ingestion of Catechins. Prog Med. 2002;22:2189-2203.

4. Chen S, Osaki N, Shimotoyodome A. Green tea catechins enhance norepinephrine-induced lipolysis via a protein kinase A-dependent pathway in adipocytes. Biochem Biophys Res Commun. 2015;461:1-7.

5. Murase T, Haramizu S, Shimotoyodome A, et al. Reduction of diet-induced obesity by a combination of tea-catechin intake and regular swimming. Int J Obes. 2006;30(3):561-568.

6. Saito K. Body Fat Reducing Effect and Safety Evaluation of Long-term Consumption of Tea Containing Quercetin Glucosides in Obese Subjects. Jpn J Pharmacol. 2015;43(2):181-194.

7. Kamiya T, Takano A, Matsuzuka Y, et al. Consumption of Pueraria Flower Extract Reduces Body Mass Index via a Decrease in the Visceral Fat Area in Obese Humans. Biosci Biotechnol Biochem. 2012;76(8):1511-1517.

8. Tominaga Y. Licorice Flavonoid Oil Effects Body Weight Loss by Reduction of Body Fat Mass in Overweight Subjects. J Health Sci. 2006;52(6):672-683

9. Najima M. Visceral fat-reducing effect of supplement containing apple polyphenol and oleanolic acid in healthy Japanese-a randomized, double-blind, placebo-controlled study, Shinryo to Shinyaku. Med Cons New-Remed. 2016;53(3):237-244.

10. Nakamura F, Ishida Y, Aihara K, et al. Effect of fragmented Lactobacillus amylovorus CP1563 on lipid metabolism in overweight and mildly obese individuals: a randomized controlled trial. Microb Ecol Health Dis. 2016;27:303-312.

11. Kamiya T, Nagamine R, Sameshima-Kamiya M, et al. The Isoflavone-Rich Fraction of the Crude Extract of the Puerariae Flower Increases Oxygen Consumption and BAT UCP1 Expression in High-Fat Diet-Fed Mice. Glob J Health Sci. 2012;4(5):147-155.

12. Aoki F, Honda S, Kishida H, et al. Suppression by licorice flavonoids of abdominal fat accumulation and body weight gain in high-fat diet-Induced Obese C57Bl/6J Mice. Bio Sci Biotechnol Biochem. 2007;71(1):206214

13. Han LK, Takaku T, Li J, et al. Anti-obesity action of oolong tea. Int $J$ Obesity. 1999;23(1):98-115.

14. Kagawa K, Matsutaka H, Fukuhama C, et al. Globin digest, acidic protease hydrolysate, inhibits dietary hypertriglyceridemia and Val-Val-Tyr-Pro, one of its constituents, possesses most superior effect. Life Sci. 1996;58(20):1745-1755.

15. Toshihisa Morimoto, Toshiyuki Miyazaki, Ryuji Murayama, et al. Wheat Albumin with Amylase-inhibitory Activity Suppresses Glycemic Rise after Rice Loading in Human Subjects. Nippon Eiyo Shokuryo Gakkaishi. 1999;52(5):285-291. 
16. Seri K, Sanai K, Matsuo N, et al. L-arabinose selectively inhibits intestinal sucrase in an uncompetitive manner and suppresses glycemic response after sucrose ingestion in animals. Metabolism. 1996;45(11):1368-1374.

17. Deguchi Y. Effects of Extract of Guava Leaves on the Development of Diabetes in the $\mathrm{db} / \mathrm{db}$ Mouse and on the Postprandial Blood Glucose of Human Subjects. Nippon Nōgeikagaku Kaishi. 1998;72(8):923-931.

18. Higashikawa F, Noda M, Awaya T, et al. 5-aminolevulinic acid, a pre- cursor of heme, reduces both fasting and postprandial glucose levels in mildly hyperglycemic subjects. Nutrition. 2013;29(7-8):1030-1036.

19. Choi MS, Ryu R, Seo YR, et al. The beneficial effect of soybean (Glycine max (L.)Merr.) leaf extracts in adults with prediabetes: a randomized placebo controlled trial. Food Funct. 2014;5(7):21-30.

20. Miura T, Ueda N, Yamada K, et al. Antidiabetic Effects of Corosolic Acid in KK-Ay Diabetic Mice. Biol Pharm Bull. 2004;29(3):585-587. 